\title{
Frequency of Developmental Dental Anomalies in the Indian Population
}

\author{
Kruthika S Guttal ${ }^{a}$ \\ Venkatesh G Naikmasur ${ }^{b}$ \\ Puneet Bhargavac \\ Renuka J Bathid
}

\section{ABSTRACT}

Objectives: To evaluate the frequency of developmental dental anomalies in the Indian population.

Methods: This prospective study was conducted over a period of 1 year and comprised both clinical and radiographic examinations in oral medicine and radiology outpatient department. Adult patients were screened for the presence of dental anomalies with appropriate radiographs. A comprehensive clinical examination was performed to detect hyperdontia, talon cusp, fused teeth, gemination, concrescence, hypodontia, dens invaginatus, dens evaginatus, macro- and microdontia and taurodontism. Patients with syndromes were not included in the study.

Results: Of the 20,182 patients screened, 350 had dental anomalies. Of these, $57.43 \%$ of anomalies occurred in male patients and $42.57 \%$ occurred in females. Hyperdontia, root dilaceration, pegshaped laterals (microdontia), and hypodontia were more frequent compared to other dental anomalies of size and shape.

Conclusions: Dental anomalies are clinically evident abnormalities. They may be the cause of various dental problems. Careful observation and appropriate investigations are required to diagnose the condition and institute treatment. (Eur J Dent 2010;4:263-269)

Key words: Dental anomalies; Hyperdontia; Microdontia; Taurodontism.

- assistant Professor, Department of Oral Medicine and Radiology, SDM Dental College and Hospital, Sattur, Dharwad, India.

b Professor and Head, Department of Oral medicine and Radiology, SDM Dental College and Hospital, Sattur, Dharwad, India.

Former Postgraduate, Department of Oral medicine and Radiology, SDM Dental College and Hospital, Sattur, Dharwad, India.

d Former Professor, Department of Oral medicine and Radiology, SDM Dental College and Hospital, Sattur, Dharwad, India.

- Corresponding author: Kruthika S Guttal Department of Oral Medicine and Radiology SDM Dental College and Hospital, Sattur, Dharwad, Karnataka, India.

Phone: +918362468142 Fax: +918362467612

Email: drkruthikadyahoo.co.in

\section{INTRODUCTION}

Developmental dental anomalies are marked deviations from the normal color, contour, size, number, and degree of development of teeth. Local as well as systemic factors may be responsible for these developmental disturbances. Such influences may begin before or after birth, hence deciduous or permanent teeth may be affected. Aberrations in the normal number of teeth include supernumerary teeth (hyperdontia), i.e. excess teeth or hypodontia (teeth missing from the normal compliment) while oligodontia is a developmental absence of six or more teeth excluding the $3^{\text {rd }}$ molars.

Anomalies of shape of teeth include microdontia and macrodontia. Microdontia refers to teeth 
that are physically smaller in size than usual and macrodontia in turn refers to teeth that are physically larger in size than normal. Anomalies of shape include dens invaginatus (DI), talon cusp, dens evaginatus, gemination, fusion, root dilacerations, taurodontism, and concrescence. ${ }^{1} \mathrm{Dl}$ is an anomaly resulting from invagination in the surface of a tooth crown or rarely the root and which is lined by enamel and dentin. ${ }^{2}$ Dens evaginatus is a focal area of the crown, projecting outward and giving rise to a horn-like protuberance on the affected surface that appears as an extra cusp. ${ }^{3}$ Talon cusp is an accessory cusp usually located on the lingual surface and rarely on the facial surface of permanent or deciduous incisors. It arises from the cingulum area, or cemento-enamel junction (CEJ) of maxillary or mandibular anterior teeth, in both the primary and permanent dentition. ${ }^{4}$ Fusion and gemination have been referred to as double teeth which appear as larger than normal sized teeth. Gemination is defined as a single enlarged tooth or joined (double) tooth in which the tooth count is normal when the anomalous tooth is counted as one. ${ }^{3}$ Fusion is defined as a single enlarged tooth or joined (double) tooth in which the tooth count reveals a missing tooth when the anomalous tooth is counted as one. ${ }^{3}$

Taurodontism is a developmental anomaly of molar teeth in which the body of the affected teeth is very large and the associated roots are shortened, with bifurcation near the apex. ${ }^{3}$ Concrescence is the union of two adjacent teeth by cementum. ${ }^{5}$ Dilaceration is an abnormal bend in the root or crown of a tooth. The bend is more frequent in the root but may be present anywhere along the length of the tooth. ${ }^{3}$

These anomalies not only affect the esthetic appearance of teeth but also pose difficulties during dental treatment and sometimes are the cause of dental problems. The present study was performed to evaluate the frequency of occurrence of dental anomalies of size, number, and shape in the adult population and their implications in the treatment of such conditions.

\section{MATERIALS AND METHODS}

This prospective study was conducted during the period from December 2004 to November 2005 and was composed of both clinical and radiographic examinations. All patients attending the outpatient department were screened for the presence of anomalies. A comprehensive clinical examination was carried out to identify the presence of hyperdontia, hypodontia, talon cusp, fused teeth, gemination, concrescence, dens invaginatus, dens evaginatus, macro- and microdontia and taurodontism.

Radiographs such as intra-oral periapical radiographs, orthopantomographs, and occlusal radiographs were advised if the condition demanded. In addition, radiographs referred to the radiology section were also examined for the presence of anomalies.

\section{Exclusion criteria}

The following groups were excluded from the study:

- Patients belonging to the pediatric age group (under the age of 14 years).

- Patients with syndromes such as Down's syndrome, ectodermal dysplasia, etc.

- Patients having cleft lip and palate.

- Dental anomalies secondary to structure, i.e. hypoplasia secondary to amelogenesis imperfecta, dentinogenesis imperfecta, or dental fluorosis.

\section{RESULTS}

A total of 20,182 patients were screened and of these, 350 patients had dental anomalies. This group was composed of 201 (57.43\%) male patients and 149 (42.57\%) females. The distribution of various anomalies among male and female patients is outlined in Table 1. Hyperdontia was the most common dental anomaly followed in descending order by root dilacerations, taurodontism, microdontia, hypodontia, talon cusp, fusion, dens evaginatus, dens invaginatus, concrescence, gemination and macrodontia. The distribution of various anomalies is outlined in Figure 1.

\section{DISCUSSION}

Dental anomalies of developmental origin reflect a change either in the number, size, shape, or location of teeth, or structural changes.

\section{Hyperdontia}

Various reports of hyperdontia are mostly in children or the adolescent population. ${ }^{6}$ Its prevalence ranges from 0.1 to $3.8 \%{ }^{6}$ but it is increasingly common in patients with cleft lip and cleft 
palate. Salcido-garcía et al have reported a higher prevalence in males than in females. ${ }^{6}$ This was also noted in the present study. Among the various supernumerary teeth, mesiodentes are the most commonly found followed by $4^{\text {th }}$ molars, premolars and maxillary lateral incisors. ${ }^{6}$ In the present study, there was a higher occurrence of paramolars followed by mesiodentes and supernumerary teeth in the anterior region. Impacted supernumerary teeth had equal occurrence in maxilla and mandible and occurred in the anterior rather than the posterior region.

\section{Hypodontia}

This occurs in permanent dentition in $0.3 \%$ of the population. ${ }^{7}$ In the present study, it was noted that missing teeth constituted $10.6 \%$ of all of the anomalies. Congenitally missing teeth most commonly occur with maxillary laterals, $2^{\text {nd }}$ premolars and mandibular central incisors and can be unilateral or bilateral. Radiographs confirm the absence of missing teeth. The etiology is believed to be hereditary or developmental. In this study, mandibular central incisors dominated the list compared to premolars and it was noted more in females. Missing third molars were not considered in the study sample.

Treatment generally requires a multidisciplinary approach including orthodontic correction, or prosthetic replacement with a removable or fixed appliance. Age of the patient, number of missing teeth, carious teeth, and condition of supporting tissues, occlusion and interocclusal space are the important factors determining treatment planning.

Hypodontia and hyperdontia were not noted concomitantly in the present study unlike the re-

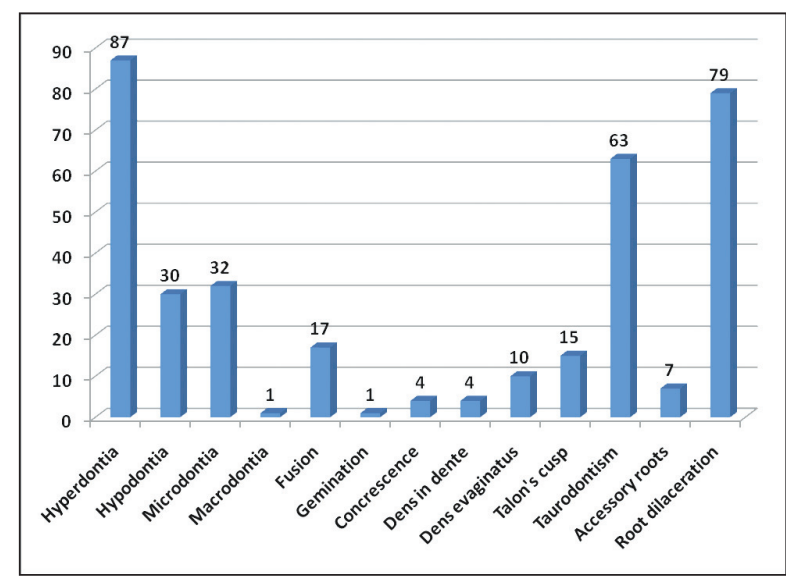

Figure 1. Distribution of various dental anomalies. port of Ranta ${ }^{8}$ where supernumerary teeth and agenesis of teeth were observed simultaneously more often in permanent dentition than in deciduous dentition.

\section{Microdontia}

Teeth commonly affected are maxillary lateral incisors and third molars. The prevalence of this condition ranges from $0.8 \%$ to $8.4 \%$ in various populations. ${ }^{1}$ When lateral incisors are affected, there is a reduction in mesiodistal diameter and convergence towards the incisal edge and this is referred to as peg shaped incisors. The condition is largely of genetic origin. In the present study, microdontia constituted $9.14 \%$ of total anomalies with a majority of peg shaped lateral incisors.

\section{Macrodontia}

Typically only a few teeth are larger than normal. Diffuse true macrodontia is observed in pituitary gigantism and pineal hyperplasia. ${ }^{1}$ In this study, only one patient presented with macrodontia affecting the maxillary central incisors.

\section{Dens invaginatus}

This presents clinically as pit or fissure on the lingual surfaces of anterior teeth. The classical radiographic appearance of coronal $\mathrm{DI}$ is as a pear shaped invagination of enamel and dentine with a narrow constriction at the opening on the surface of the tooth. ${ }^{9}$ The infolding of the enamel lining is more radiopaque than the surrounding tooth structure aiding easy identification. The crown is almost always malformed if the coronal invagination is extensive.

Oehlers et al $^{9}$ grouped coronal DI into three types according to radiographic appearance:

1. Enamel lined invaginations confined to the crown of the tooth;

2. Extending towards the root but not crossing the cemento-enamel junction (CEJ);

3. Penetrating the surface of the root and 'bursting' apically or laterally to produce a second foramen in the root.

The majority of cases are located in maxilla and in lateral incisors. When affecting the maxillary lateral incisors, the incidence among several population groups ranged from 0.25 to $5.1 \% .{ }^{9}$ Following the maxillary lateral incisors, in decreasing order, the central incisors, premolars, canines 
and molars are generally affected. DI involving mandibular teeth is rare. In the present study, DI accounted for $1.14 \%$ of anomalies and types 1 and 2 were noted with maxillary lateral incisors. There was a higher prevalence in male patients.

The possibility of the pulp being affected without a clinically detectable lesion as a result of tortuous lingual anatomy makes DI clinically significant ${ }^{9}$ since the enamel is thin and close to the pulp, and so there can be easy involvement. Pulp sensitivity testing has to be performed if radiographically apical periodontium is unremarkable in clinically suspicious teeth. If the tooth is vital then it should be restored to prevent access of the $\mathrm{Dl}$ to the oral environment. Steffen et $\mathrm{al}^{10}$ have stated that if no entrance to the invagination can be detected and there are no signs of pulp pathology, no treatment is required other than fissure sealing or a minimally invasive filling. Awareness of this anomaly when present is essential especially in a case of pulpitis in the absence of any history of trauma or clinical evidence of caries or restorations. DI with pulpal involvement is usually managed by conventional endodontic treatment or special endodontic techniques capable of inducing an apexification if the condition necessitates. Surgical treatment should be considered in cases of endodontic failure and in teeth which cannot be treated non-surgically due to anatomical problems or failure to gain access to all parts of the root canal. ${ }^{10}$

\section{Talon cusp}

This is composed of normal enamel and dentin

Table 1. Distribution of dental anomalies in male and female patients.

\begin{tabular}{lcc}
\hline Anomalies & Males & Females \\
\hline Hyperdontia & 59 & 28 \\
Hypodontia & 12 & 18 \\
Microdontia & 14 & 18 \\
Macrodontia & 13 & 1 \\
Fusion & 1 & 4 \\
Gemination & 1 & \\
Concrescence & 4 & 3 \\
Dens in dente & 9 & 1 \\
Dens evaginatus & 5 & 10 \\
Talon's cusp & 34 & 29 \\
Taurodontism & 4 & 3 \\
Accessory roots & 45 & 34 \\
Root Dilaceration & & \\
\hline
\end{tabular}

containing varying extensions of pulp tissue. It may connect with the incisal edge to produce a T-form or, if more cervical, a Y-shaped crown contour. It is also known by other names such as interstitial cusp, tuberculated premolar, evaginated odontoma, occlusal enamel pearl, occlusal anomalous tubercle or supernumerary cusp. ${ }^{4}$ Hattab et $\mathrm{al}^{4}$ classified anomalous cusps into three types based on the degree of cusp formation and extension:

1. Talon: A morphologically well-delineated additional cusp that prominently projects from the palatal surface of a primary or permanent anterior tooth and extends at least half the distance from the CEJ to the incisal edge.

2. Semi talon: An additional cusp of $1 \mathrm{~mm}$ or more, but extending less than half the distance from the CEJ to the incisal edge. It may blend with the palatal surface or stand away from the rest of the crown.

3. Trace talon: An enlarged or prominent cingula in any of its variants (i.e. conical, bifid or tubercle-like) originating from the cervical third of the root.

Its prevalence ranges from less than $1 \%$ to $8 \%$ of the population ${ }^{11}$ with a higher frequency in males than females. The anomaly has a greater predilection for the maxilla, and maxillary lateral incisors are commonly affected in the permanent dentition followed by central incisors and canines. In the present study, this anomaly accounted for $4.28 \%$ of dental anomalies and the semi-talon form was observed.

Juan et $\mathrm{al}^{4}$ have emphasized that early diagnosis and management of talon cusp is important to prevent complications such as occlusal interference, compromised esthetics, caries and periapical pathologies, and periodontal problems. Prevention of accidental cusp fracture and attrition has also been stressed.

\section{Dens evaginatus}

This is a relatively rare dental anomaly and primarily affects the premolars but can also occur on molars, canines, and incisors. In premolars and molars, the anomaly is usually seen on the occlusal surface, while in canines and incisors, it arises from the cingulum area of the lingual or palatal surface. ${ }^{3}$ In the present study, dens evaginatus comprised $2.85 \%$ of the total dental anomalies. It is clinically significant as it can lead to occlusal in- 
terference and subsequent loosening or displacement of the involved tooth. ${ }^{3}$ The tooth may remain asymptomatic for a considerable period of time. ${ }^{3}$

\section{Taurodontism}

Teeth affected by this dental anomaly present with elongated pulp chambers and apical displacement or bifurcation or trifurcation of the roots. This leads to a greater apicoocclusal height and lack of constriction at the level of the CEJ. ${ }^{2}$ So the distance from the bifurcation of roots to the CEJ is greater than the occluso-cervical distance. Taurodontism may be unilateral or bilateral and affects permanent teeth more frequently than primary teeth. It is commonly observed among the Eskimos and Natives of Australia and Central America. Taurodontism may be classified as mild, moderate or severe (hypo, meso and hyper, respectivelyl based on the degree of apical displacement of the pulpal floor. ${ }^{2}$ Mandibular molars are found to be affected more often than maxillary molars. Its prevalence has been reported to range between $5.67 \%$ and $60 \%$ of subjects. ${ }^{12,13}$ In the present study, it accounted for $18 \%$ of all of the anomalies.

As a taurodont shows wide variation in the size and shape of the pulp chamber with varying degrees of obliteration and canal configuration, root canal therapy becomes a challenge.

\section{Fusion and gemination}

These anomalies are also referred to as double teeth, formed as result of total or partial union in dentin and possibly their pulps. They are known to occur in both deciduous and permanent dentitions. $^{2}$

Fusion may be partial or complete and may present with two independent root canals or less often, a single root and one or two pulp chambers. ${ }^{14}$ As a result, the tooth may be of normal size or larger than normal. Fusion of central incisors and canines is more frequent than that of lateral incisors and canines. The prevalence ranges from $0.5 \%$ to $5 \%$ based on geographic, racial or genetic factors. ${ }^{14}$

Gemination is an incomplete division of one tooth germ, resulting in the formation of two partially or completely separated crowns formed on a single root. ${ }^{2}$ It is more frequent in the anterior teeth, but can also affect molars and bicuspids. It has a prevalence of $0.5 \%$ and $0.1 \%$ in deciduous and permanent dentitions, respectively. ${ }^{15}$

In the present study, fusion accounted for $4.85 \%$, and gemination constituted $0.28 \%$ lonly one patient) of all of the dental anomalies. Fusion was observed to occur unilaterally in accordance with other studies. ${ }^{15}$ Mandibular teeth were affected more than maxillary. Fusion can be suspected when the number of teeth in the arch is found to be reduced and/or two roots are seen radiographically. ${ }^{2}$

Double teeth will appear similar clinically and are larger than normal teeth, but by definition fusion must involve dentin. ${ }^{2}$ Gemination can usually be distinguished from fusion by the presence of a full compliment of teeth and an incompletely divided tooth.

Double teeth may adversely affect esthetics, and may lead to dental crowding and difficulty in eruption of adjacent teeth. Treatment consists of managing asymmetry, either by extirpation of the unwanted dental portion in conjunction with root canal therapy, or restoration of the exposed area. Orthodontic intervention completes the treatment plan.

\section{Accessory roots}

These are commonly known to occur in mandibular canines, premolars and molars loften in $3^{\text {rd }}$ molars). ${ }^{16}$ There are no reported studies on the prevalence or occurrence of accessory roots in different populations except for individual case reports. ${ }^{16}$ In the present study, accessory roots were noted in mandibular premolars and $1^{\text {st }}$ molars with higher numbers in males and comprising $2 \%$ of the total anomalies. This abnormality is a definite hindrance for successful endodontic treatment leading to perforations and also poses difficulty in extractions. The radiographic signs of accessory roots include double periodontal spaces on one side of the root, periodontal space crossing roots and abrupt diminution of the root canal spaces. ${ }^{16}$

\section{Concrescence}

The incidence of concrescent teeth is reported to be highest in the posterior maxilla. In the present study, only four patients had concrescence constituting $1.4 \%$ of all of the dental anomalies. It may influence surgical procedures, periodontal, endodontic and even orthodontic treatment. ${ }^{5}$ 


\section{Dilacerations}

These are thought to arise secondary to trauma during tooth formation, altering the angle between the tooth germ and the portion of the tooth already developed. ${ }^{2}$ Occasionally, the bend is created by pressure from adjacent cysts, tumors or odontogenic hamartoma. Frequently, the affected teeth are the maxillary incisors followed by the mandible anteriors. In the present study, dilacerations of the roots comprised $22.5 \%$ of the anomalies. It was noted more in males than in females. The severity of angulation seems to be related to the age of the patient, and the direction and degree of the force applied. It may produce delayed eruption or difficulties during root canal therapy or extraction. Early recognition on preoperative radiographs will minimize the problems.

A clinician may come across dental anomalies of developmental origin in day-to-day practice. They may present as an isolated abnormality or as part of various syndromes. The possible etiological factors are unclear and may be associated with varied dental problems. The current study tries to ascertain the frequency of developmental dental anomalies in the adult population unlike other studies which have evaluated the prevalence of dental anomalies in children. ${ }^{8-20}$

Ooshima et $\mathrm{al}^{21}$ have stressed that anomalies of number, size and morphology should be studied as a group rather than in an isolated fashion. To our knowledge, this is the first study to evaluate dental anomalies of number, size and shape in an adult population.

\section{CONCLUSIONS}

The present study attempts to evaluate the frequency of occurrence of various developmental dental anomalies in a non-syndromic adult Indian population. It was noted that anomalies were more frequent in male patients than in females. Hyperdontia, root dilaceration, peg shaped laterals (microdontial, and hypodontia were more frequent compared to other anomalies of size and shape.

Developmental anomalies of teeth are clinically evident abnormalities. They may be the cause of various dental problems. Careful observation and appropriate investigations are required to diagnose the condition and institute appropriate treatment.

\section{REFERENCES}

1. Neville DW, Damm DD, Allen CM, Bouquot JE. Abnormalities of teeth. In: Oral and Maxillofacial Pathology. 2nd ed. Philadelphia, PA: Elsevier; 2005:49-89.

2. Neville DW, Damm DD, Allen CM, Bouquot JE. Color Atlas of Clinical Oral Pathology. 2nd ed. Baltimore, MD: Williams and Wilkins; 1991:62-64.

3. Vasudev SK, Goel BR. Endodontic management of dens evaginatus of maxillary central incisors: A rare case report. J Endod 2005;31:67-70.

4. Juan JS, Jiménez-Rubio A. Talon cusp affecting permanent maxillary lateral incisors in 2 family members. Oral Surg Oral Med Oral Pathol Oral Radiol Endod 1999;88:90-92.

5. Romito LM. Concrescence: report of a rare case. Oral Surg Oral Med Oral Pathol Oral Radiol Endod 2004;97:325-327.

6. Salcido-garcía JF, Ledesma-montes C, Hernández-flores F, Pérez D, Garcés-ortíz M. Frequency of supernumerary teeth in Mexican population. Med Oral Patol Oral Cir Bucal 2004;9:403-409

7. Akkaya N, Kiremitçi A, Kansu Ö. Treatment of a patient with oligodontia: A case report. J Contemp Dent Pract 2008;3:121-127.

8. Ranta R. Numeric anomalies of teeth in concomitant hypodontia and hyperdontia. J Craniofac Genet Dev Biol 1988;8:245-251.

9. Mupparapu M, Singer SR. A rare presentation of dens invaginatus in a mandibular lateral incisor occurring concurrently with bilateral maxillary dens invaginatus: Case report and review of literature. Aust Dent J 2004;49:90-93.

10. Steffen H, Splieth C. Conventional treatment of dens invaginatus in maxillary lateral incisor with sinus tract: One year follow-up. J Endod 2005;31:130-133.

11. Dash JK, Sahoo PK, Das SN. Talon cusp associated with other dental anomalies: a case report. Int J Paediatr Dent 2004; 14:295-300.

12. Darwazeh AM-G, Hamasha AA-H, Pillai K. Prevalence of taurodontism in Jordanian dental patients. Dentomaxillofac Radiol 199;27:163-165.

13. Ashwin R, Arathi R. Taurodontism of deciduous and permanent molars: Report of two cases. J Indian Soc Pedod Prev Dent 2006;24:42-44.

14. Oliván-rosas G, López-jiménez J, Giménez-prats MJ, Piqueras-Hernández M. Considerations and differences in the treatment of a fused tooth. Med Oral 2004;9:224-228.

15. Hernández-Guisado JM, Torres-Lagares D, Infante-Cossío P, Gutiérrez-Pérez JL. Dental gemination: report of case. Med Oral 2002; 7:231-236.

16. Arslan A, Ozel E, Altundal H. Various root abnormalities. Report of three cases. NY State Dent J 2008;74:41-43. 
17. Clayton JM. Congenital dental anomalies occurring in 3557 children. J Dent Child 1956;23:206-208.

18. Buenviaje TM, Rapp RD. Dental anomalies in children: a clinical and radiographic survey. J Dent Child 1984;51:4246.

19. Osuji 00, Hardie J. Dental anomalies in a population of Saudi Arabian children in Tabuk. Saudi Dent J 2002;14:1114.

20. Cho SY, Ki Y, Chu V, Chan J. Concomitant developmental dental anomalies in Chinese children with dens evaginatus. Int J Paediatr Dent 2006;16:247-251.

21. Ooshima T, Ishida R, Mishima K, Sobue S. The prevalence of developmental anomalies of teeth and their association with tooth size in the primary and permanent dentitions of 1650 Japanese children. Int J Paediatr Dent 1996;6:87-94. 